\title{
An Analysis of Cohesion Achieved by Imperative Clause in English Netvertising
}

\author{
Junhua Ren \\ Foreign Language College, Tianjin Polytechnic University, Tianjin, China \\ Email: renjunhua2010@126.com
}

\begin{abstract}
The present study finds that imperative clause, which is ubiquitous in English netvertising, aids to make the discourse of a net ad cohesive in two ways: non-structure and structure. The former consists of reference, ellipsis and lexical cohesion. The latter refers to the chain of imperative clauses that forms a relation of co-classification.
\end{abstract}

Index Terms - imperative clause, English netvertising, cohesion

\section{INTRODUCTION}

\section{A. Imperative Clause in English Netvertising}

The word, 'advertising', first appeared in a magazine in 1645 and it originates in Latin 'advertere' which means 'turning towards' while it means 'speaking publicly' in Chinese and 'shouting or yelling' in Russian. The history of advertising is not very long but the definitions of advertising offered by different scholars are various (Hu, 1996).It is estimated that an average person in the world will get involved in advertisements for 800 times a day (Qi, 2003). Advertising surrounds us and there is no reason for us to be elusive from it.

Generally speaking advertising has the following functions: information function, demand creation function, persuasive function, get action function and goodwill establishment function (Wang, 2004). In order to fulfill the above functions the copywriter should keep the four principles of AIDA advanced by E. St. Elmo who was the Advertising Manager for NCR and served as Vice President at Campbell-Ewald Company. AIDA is the abbreviation of Attention, Interest, Desire and Action. Qi Yunfang (2003) adds other three principles: Conviction, Memory and Satisfaction. He thinks that the copywriter should pay attention to the two values of English advertising: Attention value and Memory value. The two values promulgate that English advertising is a unique discourse.

According to different media, advertising can be classified into various types: billboards, radio, cinema and television, Internet, sky-writing, bus stop benches, magazines, newspapers, sides of buses, taxicab doors and the backs of event tickets etc. Among them, advertising by adopting the medium of Internet, netvertising for short, has some advantages that traditional advertising is short of. The advantages of netvertising are low cost, targetability, tracking, flexibility and interactivity. Because of these advantages more and more companies view netvertising as a real opportunity. Due to this net advertisements (net ads) are chosen as the corpus for the present study. 124 English classified advertisements are downloaded from three different websites: Overstock, deal and rEdenvelop. From the 124 net ads, 50 are chosen randomly. In the 50 net ads one type of clause is pervasive, which is imperative clause. 121 imperative clauses are identified in the 50 net ads. Such quantity means that more than two imperative clauses used in each net ad. Therefore the ubiquity of imperative clause in English netvertising, a particular discourse, makes it possible to study the cohesion achieved by this type of clause in English netvertising.

\section{B. Cohesion}

In Halliday and Hasan's term cohesion is semantic. It occurs where the interpretation of some element in the discourse is dependent on that of another. Cohesion in Halliday and Hasan's book Cohesion in English refers specifically to the non-structural text forming relations. However they expand the scope of the concept of cohesion in another book Language, Context and Text, which covers both non-structural and structural text forming relations (Zhang, 2003).

Non-structural cohesion mainly refers to grammatical cohesion and lexical cohesion. The former includes reference, substitution and ellipsis; the latter refers to reiteration and collocation. There is a borderline between the two types of cohesion, which is conjunction (Halliday and Hasan, 2001). Structural cohesion consists of thematic structure, information structure, transitivity and mood structure. In this study it is found non-structural and structural cohesion can be achieved by imperative clause in English netvertising. The former refers to reference, ellipsis and lexical cohesion; the latter is realized by the chain of imperative clauses in English netvertising.

\section{COHESION ACHIEVED BY IMPERATIVE CLAUSE IN ENGLISH NETVERTISING}

\section{A. Reference}


Reference is a semantic relation and it can be further divided into exophora and endophora. Halliday thinks that exophora links the language with the context of situation, so it does not contribute to the integration of one passage and only endophora is cohesive (ibid), but Delu Zhang (2003) claims that exophora is also cohesive because it contributes to cohesion implicitly and the referential item can be retrieved from the context of situation or the context of culture. There are three types of reference: personal, demonstrative and comparative reference (Halliday and Hasan, 2001).The present study finds Exophoric personal reference is widely used in imperative clause in netvertising. See the following examples:

(1) keep out the cold with Marmot's Lassen Wind Stopper fleece vest

(2) send a beautiful message of Love, Hope, and Faith with this inspirational multi colored bracelet

(3) outfit your car with the sweet styling and functionality of this in dash DVD/CD stereo from SPL

(4) let your hair hang free to gracefully play with the sparkle of the earrings

The subjects of the four imperative clauses are implicit 'you' which refer to the audiences. 'your' appearing in (3) and (4) also stands for the audiences. Obviously the implicit subject 'you' and the possessive pronoun 'your' belong to exophorical personal reference which connects the audiences with the advertisements closely. The audiences are certain to be persuaded by the advertisers to have a try of the products when they read such imperative clauses.

Exophoric demonstrative reference can also be found in imperative clauses in the net ads. Examples are illustrated below.

(5) maintain your court sense with this hybrid running shoe from Fila

(6) shed light on your studies or leisure reading with this classically styled desk lamp

(7) keep your toes cozy warm in these trendy and practical 'snow' boots from Chinese Laundry

(8) order your new ring now at our low online price

(9) order now to take advantage of this great one-of-kind deal

'this' in (5) and (6) and 'these' in (7) are near demonstrative reference. 'this' conveys a sense of immediacy and also of solidarity with the hearer, of shared interest and attention (Halliday, 2000), so it is frequently used in the net ads to bring out the products for catching the attention of the audiences. 'this' and 'these' are exophoric in the above imperative clauses for they refer to the products in the pictures which can be understood as a type of context (Thibault, 2004). 'now' in (8) and (9) refers exophorically to the current time when the audiences are reading the advertisements and it is used to persuade the audiences to choose or to buy the products at the time when they are browsing the web pages.

Endophora used in imperative clause is also traceable in netvertising. The following examples are extracted from three net ads in the 50 ones.

(10) wow them with 6 chocolate bars $(1.25 \mathrm{oz})$

(11) simply roll it up

(12) top it off with a USB connection cable for fast downloads

(13) read more

'them' and 'it' in (10), (11) and (12) are anaphoric; 'more' in (13) is cataphoric. 'them' in (10) refers to people who have a sweet tooth, being mentioned in the previous part of the advertisement; 'it' in (11) is the raised air bed also appearing in the former part of the advertisement; 'it' in (12) stands for the RCA Lyra personal digital player being described at the very beginning of the net ad; 'more' in (13) is comparative cataphoric. The content locates in the latter link.

Exophora and endophora in imperative clause contribute jointly to the cohesion of netvertising discourse.

\section{B. Ellipsis}

Ellipsis can be interpreted as that form of substitution in which the item is replaced by nothing. There are nominal ellipsis, verbal ellipsis and clausal ellipsis. For the sake of not being redundant, ellipsis is used in imperative clauses in the net ads but it is not as pervasive as reference. See the following examples:

(14) order one for a quiet retreat among the flowers or order several for outdoor entertaining

(15) get one for yourself as well, at our low online price

In (14) the nominal group 'Brazilian hardwood adirondack ottoman' following 'one' and 'several' is omitted; in (15) the elliptical element after 'one' is 'sterling silver heart toggle bracelet'. The omitted elements can be retrieved from the previous parts of the advertisements.

\section{Lexical Cohesion}

In lexical cohesion reiteration involves the use of repetition, synonym, a near synonym and superordinate. Collocation refers to the co-occurrence of lexical items. Reiteration of lexical cohesion is traced in imperative clause in netvertising discourse. Examples are below.

(16) Experience the health benefits obtained by walking naturally with the help of Energy sandal from Earth shoes. Find great deals on shoes for the entire family at Overstock.com.

(17) Treat your guests with the delicious aroma and robust flavor of Café Godiva.... It's a delicious way to start the day or to end a fine meal.

(18) Save $50 \%$ on a second box (to the same address) of the sweetest fruit to bend a bough.... Save on two luscious 
boxes (a $\$ 55.90$ value), shipped to one address.

(19) Order now to get in line. Hurry, order now and we'll ship this item when it becomes available.

'shoes' in (16) appearing in the two clauses is repetition. It is also the superordinate of 'sandal' appearing in the first clause. The adjective 'delicious' and the verbs 'save' and 'order' all appear twice in the two clauses in (17), (18) and (19) respectively. They all belong to repetition.

Besides the non-structural cohesion: reference, ellipsis and lexical cohesion, the structural cohesion can also be realized by imperative clause in netvertising. Delu Zhang (2003) studies cohesive function of mood structure and argues that the high frequency of one mood type in a discourse represents the prominent characteristic of an interpersonal meaning. In his opinion there is a relation of co-classification between the same clause types, which is cohesive. Imperative clause is pervasive in netvertising and such a high frequency of imperative clause builds a cohesive tie for the netvertising discourse. See the following DVD/VCR net ad.

\section{JVC Progressive Scan DVD/VCR Combo}

Looking for a replacement VCR? Want a new DVD player? The HR-XVC33 provides the logical choice for people who want the best of both worlds.

Enjoy a variety of media. Watch a DVD while recording a TV program on tape. Even dub DVD to VCR (non-copyrighted material only). With the HR-XVC33, it's not only possible; it's easy as JVC.

Click on Overstock.com for hot deals on the biggest names in electronics.

In the above net ad the imperative clauses in italic are like a chain and they help to consolidate the cohesion of the discourse and offer some information of the product for audiences. They also form a strong sense of persuasion which influences audiences to make a decision of choosing or buying the products. There is more than one among the 50 net ads that contain such a chain of imperative clauses.

Further reading the above net ad we can easily find the two clauses in ellipsis appearing at the very beginning of the discourse are interrogatives. The two interrogatives also contribute to the cohesion of the discourse because they share the same mood type. Actually they are only interrogative clauses literally for the meaning of the two clauses is to persuade audiences to have a new DVD player, HR-XVC33 specifically, instead of asking a question, so they can be understood as imperative in the net ad, which will not be analyzed concretely in the present study.

\section{CONCLUSION}

Imperative clause is mainly used for the meaning of persuasion which is an important function of English netvertising since net ads aim to persuade the audiences to buy or to enjoy the products. Therefore imperative clause is ubiquitous in English netvertising. The present study focuses on the cohesion achieved by imperative clause in English netvertising. It is found non-structural and structural cohesion are achieved by imperative clause in netvertisng. The former includes reference, ellipsis and lexical cohesion. The latter refers to the chain of imperative clauses that forms a relation of co-classification.

\section{ACKNOWLEDGMENT}

The author wishes to thank Professor Zhan and Sun who work in Beihua University. With their patient guidance the author reads more books about functional grammar, which are very important for the author to study the imperative clause in English netvertising.

\section{REFERENCES}

[1] Halliday, M. A. K. (2000). An Introduction to Functional Grammar (2 ${ }^{\text {nd }}$ edition). Beijing: Foreign Language Teaching and Research Press.

[2] Halliday, M. A. K. (2001). Language as Social Semiotic: The Social Interpretation of Language and Meaning. Beijing: Foreign Language Teaching and Research Press.

[3] Halliday, M. A. K. \& Hasan, R. (2001). Cohesion in English. Beijing: Foreign Language Teaching and Research Press.

[4] Halliday, M. A. K. (2002). Linguistic Studies of Text and Discourse. London: Continuum.

[5] Hu, Rui. (1996). Modern Advertising. Zhejiang: Zhejiang University Press.

[6] Qi, Yunfang. (2003). Advertising and English Advertising. Zhejiang: Zhejiang University Press.

[7] Thibault, P.J . "The News Story Genre." Lecture at Lecture Hall 702. School of Foreign Languages Northeast Normal University, ChangChun, JiLin 8 Apri. 2004.

[8] Wang, Yanxi. (2004). English Advertising. Beijing: University of International Business and Economics Press.

[9] Zhang, Delu. (2003). The Development of the Theory of Text Coherence and Cohesion and Its Applications. Shanghai: Shanghai Foreign Language Education Press.

Junhua Ren is a lecture in Foreign Language College of Tianjin Polytechnic University. She has taught the students the following courses: College English, Interpretation and Business Negotiation etc. Her research interests are functional grammar, discourse analysis and SLA. 